\title{
Relapse of Lymphangioleiomyomatosis Five Years after Bilateral-Lung Transplantation
}

\author{
Jalal Heshmatnia, MD'; Maryam Sadat Mirenayat, $\mathbf{M D}^{1,2^{*}}$; Mitrasadat Rezaei, $\mathbf{M D}^{3,4}$; Felix Bongomin, $\mathrm{MD}^{5}$; Mehrdad \\ Bakhshayeshkaram, $\mathbf{M D}^{6}$; Payam Tabarsi, $\mathbf{M D}^{7}$; Kambiz Sheikhy, $\mathbf{M D}^{8}$; Vida Mortezaee, $\mathbf{P h D}^{1}$ \\ ${ }^{1}$ Chronic Respiratory Diseases Research Center, National Research Institute of Tuberculosis and Lung Diseases (NIRTLD), Shahid \\ Beheshti University of Medical Sciences, Tehran, Iran \\ ${ }^{2}$ Lung Transplantation Research Center (LTRC), National Research Institute of Tuberculosis and Lung Diseases (NIRTLD), Shahid \\ Beheshti University of Medical Sciences, Tehran, Iran \\ ${ }^{3}$ Pathology Department, School of Medicine, Shahid Beheshti University of Medical Sciences, Tehran, Iran \\ ${ }^{4}$ Virology Research Center (VRC), National Research Institute of Tuberculosis and Lung Diseases (NIRTLD), Shahid Beheshti \\ University of Medical Sciences, Tehran, Iran \\ ${ }^{5}$ Division of Infection, Immunity and Respiratory Medicine, Faculty of Biology, Medicine and Health, The University of Manchester, \\ Manchester, UK \\ ${ }^{6}$ Pediatric Respiratory Diseases Research Centre (PRDRC), National Research Institute of Tuberculosis and Lung Diseases (NIRTLD), \\ Shahid Beheshti University of Medical Sciences, Tehran, Iran \\ ${ }^{7}$ Clinical Tuberculosis and Epidemiology Research Center, National Research Institute of Tuberculosis and Lung Diseases (NIRTLD), \\ Shahid Beheshti University of Medical Sciences, Tehran, Iran \\ ${ }^{8}$ Tracheal Diseases Research Center (TDRC), National Research Institute of Tuberculosis and Lung Diseases (NIRTLD), Shahid \\ Beheshti University of Medical Sciences, Tehran, Iran
}

\begin{abstract}
Pulmonary lymphangioleiomyomatosis (LAM) is an uncommon disease principally affecting women during childbearing years and eventually leading to progressive respiratory failure. Lung transplantation is a viable option for patients with end-stage disease. LAM-related complications remain common, but recurrence of LAM following allograft transplantation is rare. We present a 25-year-old woman who presented with progressive dyspnea five years after bilateral lung transplantation for end-stage LAM. Histological examination of transbronchial lung biopsy sample confirmed recurrent LAM. We changed cyclosporine to sirolimus and she is currently being considered for re-transplantation.

Keywords: Lung, Lymphangioleiomyomatosis, Transplantation

Cite this article as: Heshmatnia J, Mirenayat MS, Rezaei M, Bongomin F, Bakhshayeshkaram M, Tabarsi P, et al. Relapse of lymphangioleiomyomatosis five years after bilateral-lung transplantation. Arch Iran Med. 2021;24(9):701-703. doi: 10.34172/ aim.2021.101
\end{abstract}

Received: November 29, 2020, Accepted: March 15, 2021, ePublished: September 1, 2021

\section{Introduction}

Lymphangioleiomyomatosis (LAM) is a rare disease that results in cystic lung destruction. The etiology of LAM is unknown; however, TSC-LAM is associated with somatic mutations in the TSC1 or TSC 2 genes, resulting in cellular proliferation and tissue invasion. ${ }^{1}$ The features of this disease are cystic destruction of the lung with progressive pulmonary dysfunction and the presence of abdominal tumors.

The proliferation of LAM cells may lead to airflow obstruction, air trapping, cystic formation, and pneumothoraces. Cuboidal epithelioid cells in the LAM lesion react with a monoclonal antibody known as human melanoma black-45 (HMB-45); since other smooth muscle-predominant lesions in the lung do not react with the antibody, this immunohistochemical marker is useful for diagnosis. ${ }^{2}$ The first pathological disorder is the proliferation of atypical smooth muscle cells that connect with lung's LAM-like cells leading to the formation of cysts. The monoclonal anti-body HMB-45 that is a classic marker for the diagnosis of LAM in lung biopsy sections can also be found in angiomyolipoma. ${ }^{3}$

The initial signs of pulmonary LAM are exertional dyspnea, pleural effusion, spontaneous pneumothorax, and chylothorax. Extra-pulmonary manifestations of LAM include renal angiomyolipoma, lymphangioma, and lymphadenopathy forms. Diagnosis is made by a combination of clinical features and computed tomography scanning or, in cases of doubt, lung biopsy.

Treatment includes supportive care, bronchodilators, and for those with progressive disease, mammalian target of rapamycin (mTOR) inhibitors. Lung transplantation is the only treatment option currently available for severe LAM. ${ }^{4}$ LAM-related post-operative complications are common and are associated with high morbidity, but recurrence of LAM following lung transplantation is uncommon.

In this report, we present an unusual case of recurrence 
of LAM five years after bilateral lung transplantation.

\section{Case Report}

In February 2011, we received a 28-year-old woman with LAM diagnosed radiologically at the age of 27 years. Lung biopsy and histology confirmed the diagnosis. Eight months prior to transplantation, sirolimus was started, but unfortunately, she developed severe chest pain that was thought to be an adverse event related to sirolimus therapy resulting into discontinuation of sirolimus after a week of therapy. She subsequently underwent bilateral lung transplantation in November 2011. There was no evidence of LAM infiltration in the kidneys or any other organ at the time of the transplantation. After lung transplantation, histological examination of the explanted lung tissue revealed multiplication of LAM cells that were positive for the monoclonal antibody, HMB-45. After lung transplantation, she received cyclosporine, prednisolone, and mycophenolate mofetil for post-transplant immunosuppression and also received cotrimoxazole for Pneumocystis jirovecii pneumonia prophylaxis, voriconazole for prophylaxis against invasive fungal infections and valganciclovir for antiviral prophylaxis.
Through continual follow-ups after lung transplantation, our patient remained stable and asymptomatic with good and stable lung volumes.

In the middle of 2018, she referred back to us with a history of worsening dyspnea. There was a significant decline in her pulmonary function. Chest CT scan was performed for the patient. Fibrosis was seen in symmetrical and bilateral and by the thickness of interlobular septa and parenchymal bands were seen in the patient's lung CT scan (Figure 1). The patient underwent bronchoscopy and transbronchial lung biopsy tissue sections were examined: Lung parenchyma with focal interstitial inflammation and thickening with one area of spindle cell proliferation highlighted on smooth muscle actin (SMA) and HMB-45 immunohistochemical staining were observed (Figure 2). With these pieces of evidence, a diagnosis of recurrent LAM was reached. She continued on her previous immunosuppressive agents except for cyclosporine that was substituted with sirolimus. At the present time, no change was seen in the patient's pulmonary function tests based on continual follow-ups and the patient is doing fairly well and under surgical consideration for retransplantation.

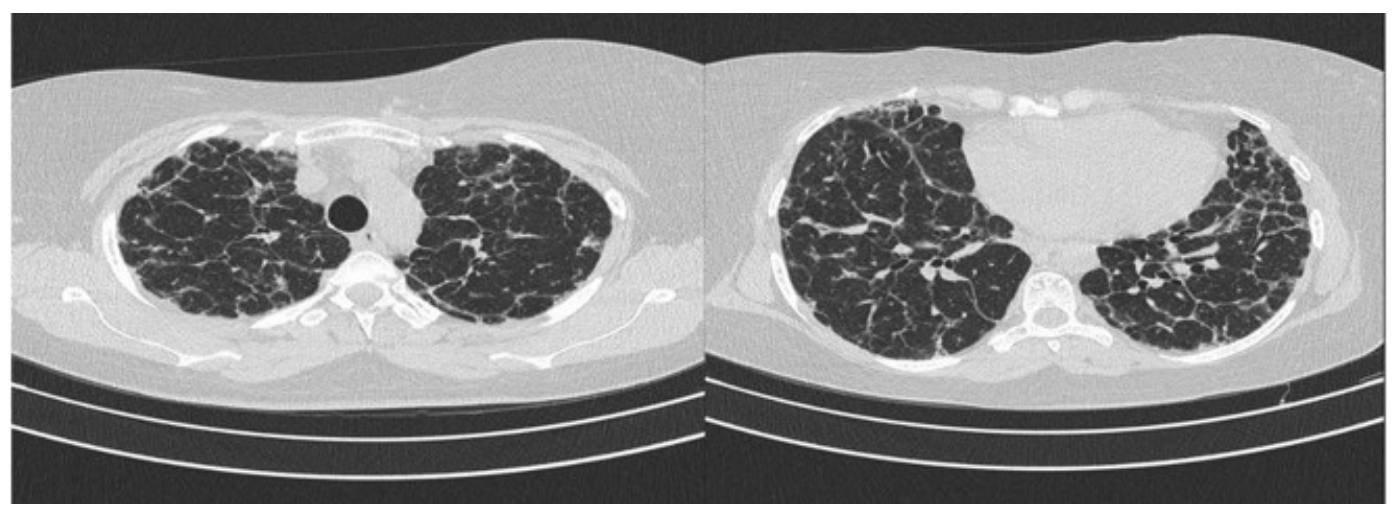

Figure 1. Diffuse Bilateral and Symmetrical Fibrosis Evidenced by Interlobular Septa Thickening and Parenchymal Bands of Fibrosis. This is a radiological evidence of a recurrent LAM.

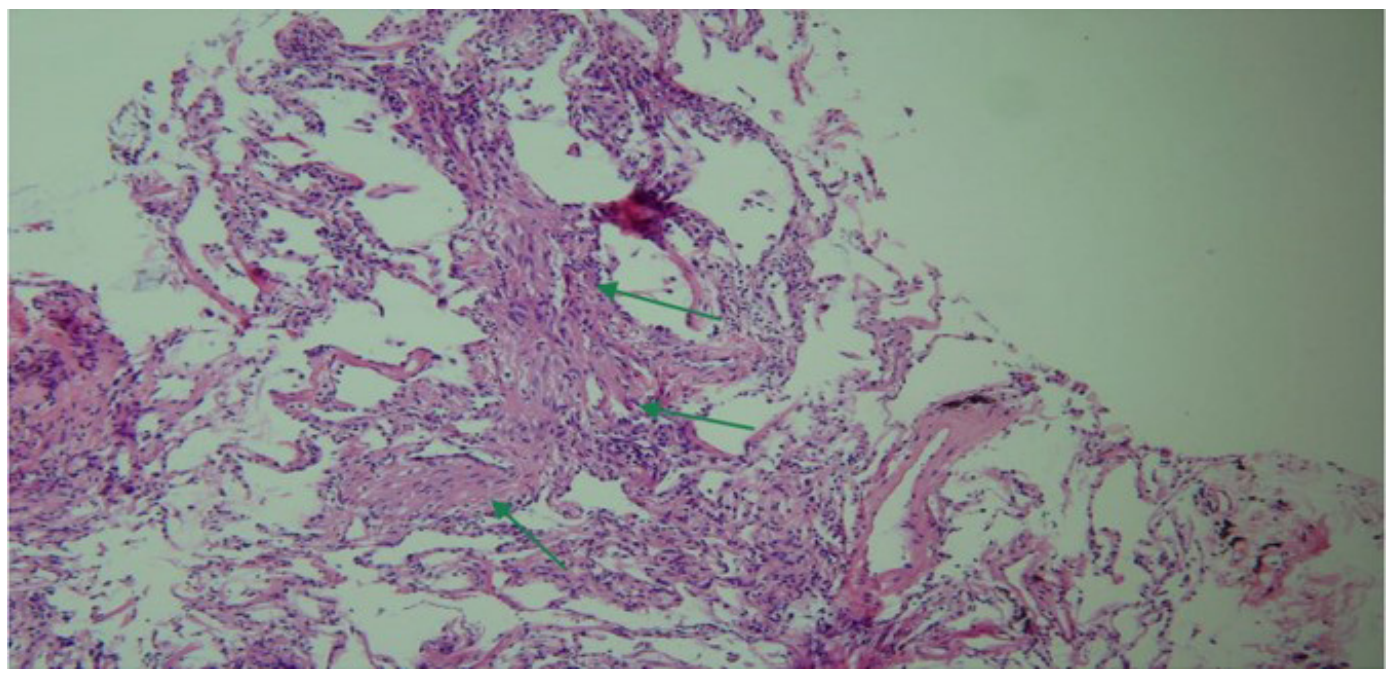

Figure 2. Lung Parenchyma with Focal Interstitial Inflammation and Thickening with One Area of Spindle Cell Proliferation Highlighted in SMA and HMB45 Immunostaining. 


\section{Discussion}

LAM is a rare systematic disease. Transplant preservation in end-stage patients who suffer from LAM is equal or even better than patients who receive a transplant for other reasons. It was found that both the lung function and quality of life ameliorated after lung transplantation in comparison to those patients who suffer from advanced disease. An improvement of $1.1 \%$ was seen following bilateral lung transplantation compared to $<0.8 \%$ in patients who received unilateral lung transplantation. ${ }^{5}$ The survival rate of patients who suffer from LAM after lung transplantation is estimated to about $60 \%-70 \% .{ }^{6}$ However, relapses of LAM after lung transplant are quite rare and the risk factors are poorly understood. To date, only 10 cases of post-transplant recurrence have been reported in patients treated for LAM by transplantation.

Treatment with sirolimus may be helpful in patients who suffer from LAM. A large multicenter study, the Italian Lung Cancer in the Elderly trial (MILES) study, supported the use of sirolimus at a dose of $2 \mathrm{mg} / \mathrm{d}$ in patients who suffer from LAM with pulmonary insufficiency - i.e. predicted FEV $1<70 \%$. $^{7}$ Moreover, sirolimus successfully treated relapse after transplantation.

Our patient has shown significant clinical improvement on sirolimus and she is now a potential candidate for re-transplantation. A case report of a successful use of sirolimus for stabilization of lung function following the recurrence of LAM disease after lung transplantation has been previously published. Lung transplantation remains one of the most acceptable therapeutic options for end-stage LAM patients with severe respiratory failure. Both single and double lung transplantation have been performed. According to large multi-center studies across Europe and the US, the recurrence of LAM after lung transplantation is uncommon with rates as low as $3.7 \%-7 \%{ }^{7}$

\section{Authors' Contribution}

$\mathrm{JH}, \mathrm{MR}$ and $\mathrm{KSH}$ performed preliminary studies. MM, VM, and $\mathrm{PT}$ evaluated the patient and wrote the initial version of the article. $\mathrm{MB}$ and FB did the final editing of the article. All authors read and approved the final version of the article.

\section{Conflict of Interest Disclosures}

The authors of this article have no conflict of interest.

\section{Ethical Statement}

This study was performed with the patient's consent and she was aware of the participation.

\section{Acknowledgements}

We thank our colleagues from Chronic Respiratory Diseases Research Center, National Research Institute of Tuberculosis and Lung Diseases (NIRTLD), Shahid Beheshti University of Medical Sciences, Tehran, Iran and Professor Hamid Reza Jamaati, Chief of Chronic Respiratory Disease Research Center (CDRC).

\section{References}

1. Johnson SR. Lymphangioleiomyomatosis. Eur Respir J. 2006;27(5):1056-65. doi: 10.1183/09031936.06.00113303.

2. Matsumoto Y, Horiba K, Usuki J, Chu SC, Ferrans VJ, Moss J. Markers of cell proliferation and expression of melanosomal antigen in lymphangioleiomyomatosis. Am J Respir Cell Mol Biol. 1999;21(3):327-36. doi: 10.1165/ ajrcmb.21.3.3693.

3. Hoon V, Thung SN, Kaneko M, Unger PD. HMB- 45 reactivity in renal angiomyolipoma and lymphangioleiomyomatosis. Arch Pathol Lab Med. 1994;118(7):732-4.

4. Johnson SR, Taveira-DaSilva AM, Moss J. Lymphangioleiomyomatosis. Clin Chest Med. 2016;37(3):389-403. doi: 10.1016/j.ccm.2016.04.002.

5. Starnes VA, Bowdish ME, Woo MS, Barbers RG, Schenkel FA, Horn MV, et al. A decade of living lobar lung transplantation: recipient outcomes. J Thorac Cardiovasc Surg. 2004;127(1):114-22. doi: 10.1016/j.jtcvs.2003.07.042.

6. Maurer JR, Ryu J, Beck G, Moss J, Lee JC, Finlay G, et al. Lung transplantation in the management of patients with lymphangioleiomyomatosis: baseline data from the NHLBI LAM Registry. J Heart Lung Transplant. 2007;26(12):12939. doi: 10.1016/j.healun.2007.09.013.

7. Zaki KS, Aryan Z, Mehta AC, Akindipe O, Budev M. Recurrence of lymphangioleiomyomatosis: nine years after a bilateral lung transplantation. World J Transplant. 2016;6(1):249-54. doi: 10.5500/wjt.v6.i1.249. 